\title{
Study the Radial Expansion of SN 1987A Using Counting Pixels Method
}

\author{
Baha T. Chiad ${ }^{1}$, Layth M. Karim², Lana T. Ali ${ }^{2}$ \\ ${ }^{1}$ Department of Physics, University of Baghdad, Baghdad, Iraq \\ ${ }^{2}$ Department of Astronomy and Space, University of Baghdad, Baghdad, Iraq \\ Email: lana_talib@yahoo.com
}

Received July 7, 2012; revised August 15, 2012; accepted August 27, 2012

\begin{abstract}
Physical properties of supernova 1987A were investigated including: Expansion velocity; Current mass; Radius; Temperature and the Rate of the expansion by applying Counting Pixels Method (CPM) in comparison with the application of the Povida and Self-Similar Solution theoretical models which in forced the idea for adopting such a model to be applied for supernova type II. Then tow results are found to be in a good agreement with the Self-Similar solution model rather than that of Povida model. The high density of the region that surrounded the exploding system acting as decelerating parameter and down the expansion velocity of the supernova from $3900 \mathrm{~km} \cdot \mathrm{s}^{-1}$ to $1200 \mathrm{~km} \cdot \mathrm{s}^{-1}$ during the past 23 years from the explosion, with a current size equal $0.39 \mathrm{pc}$, and with an expansion rate of 0.41 per a year.
\end{abstract}

Keywords: Supernovae; Individual (SN 1987A)-Supernova Remnants

\section{Introduction}

Core-collapse supernovae represent the dramatic endpoint of the life of a massive star and most of our information about it was from the theoretical hypotheses of astrophysicists; this was the case until 1987 when the nature has offered us a unique opportunity to learn the details of one of the most frequent and violent events in the universe, this was the discovery of Supernova 1987A (SN 1987A) that occurred in the Large Magellanic Cloud (LMC), a companion galaxy to our own Milky Way galaxy, on 24 February 1987 [1].

This supernova had been discovered and first formally reported by Ian Shelton a graduate student from the University of Toronto using 10-inch astrograph at the Las Campanas Observatory high in the Chilean Andes but the first visual observation made by Oscar Duhalde which was a night assistants also at the Las Campanas Observatory [2]. Moreover within the same 24 hours independent sighting came from amateur observer, Albert Jones in Nelson, New Zealand [3].

Immediately after the supernova was announced, literally every telescope in the southern hemisphere started observing this exciting new object [4], because it was the first supernova easily observable with the naked eye since the one recorded by Kepler in 1604 and the brightest for 383 years beside it is the first and the only object outside our solar system that neutrinos had been detect from it [5].
Since that time SN 1987A has taken its place not only as a unique event in modern astronomy but also as one of the most thoroughly studied objects outside the solar system that detected by instrument on the ground, below the ground, and in space, moreover it has been studied at all wavelengths from radio through gamma rays [6]. The optical study of the southern hemisphere and especially in Chili observatory confirmed that this supernova occurred in the LMC at a distance about $50 \mathrm{Kpc}$ from our Milky Way galaxy at Right Ascension $\left[\alpha=05^{\mathrm{h}} 35^{\mathrm{m}}\right.$ $\left.49.992^{\mathrm{s}}\right]$ and Declination $\left[\delta=-69^{\circ} 17^{\prime} 50.08^{\prime \prime}\right]$ [7], and it cause from the exploding of $20 \mathrm{M}_{\odot}$ Blue Supergiant star (BSG) which is called in the astronomical atlas as Sk $-69^{\circ} 202$ (for star 202 in the $-69^{\circ}$ declination band of the Sanduleak catalog) [2] and like all blue supergiant it was extremely luminous with an absolute visual magnitude of $-6.3^{\mathrm{m}}$, and with a visual magnitude $12.4^{\mathrm{m}}$ and spectral class $\mathrm{B}_{3} \mathrm{I}[3]$.

The available observations of $\mathrm{Sk}-69^{\circ} 202$, together with theoretical evolutionary model suggest that the progenitor star of SN 1987A had been a Red Supergiant (RSG) for between $10^{5}$ and $10^{6}$ years [8]; but due to the substantial mass loss before the explosion, probably as a red supergiant wind which is approximately about $8.8 \times$ $10^{-6} \mathrm{M}_{\odot}$ every year [9], its radius decreased and it evolved towards blue supergiant approximately 20,000 years before the $\mathrm{SN}$ event [10].

The astronomers have various suggestions about this transition from RSG to BSG, one of them is the low met- 
allicity of the LMC as the reason of massive star to evolves to BSG and explode rather than explode as RSG as has been expected for Type II supernova [11].

The unusual properties of this supernova deduced due to the extent of the stellar winds from both the red and blue supergiant due to this transition, such as the high density region that surround the exploding star which represented by the triple ring system that consisting of a central circular ring of diameter $0.4 \mathrm{pc}$ called equatorial ring and two other similar rings of diameter $0.9 \mathrm{pc}$ one on either side which is oriented north and south of the supernova forming an hour-glass structure [12]. This system had been observed after 310 days from the explosion by Ground-based and HST [13] through its $\lambda 5007 \AA$ [OIII] emission line [14], which emitted due to the interaction that occurred between the soft X-ray from the explosion and these stationary rings. But In 1997 HST revealed that, the first interaction between the blast wave of SN 1987A and the dense fingers that protrude inwards from the equatorial ring had been occur and it could be traced back to as early as 1995 March which means that, the blast wave started to enter into this dense region from the space [15].

In this paper, the optical images of the remnant of SN 1987A which observed by HST will be interpreted in order to measure the physical size of the remnant in addition to the overall rate of expansion, using a simple method called Counting Pixels Method in comparison with the results that obtained from applying two theoretical models, which are Povida model and Self-Similar Solution model as will preformed in the next section.

\section{Counting Pixels Method}

Counting Pixels Method is considered as the simplest method that can give the radius of any system by measuring its true linear diameter from counting their pixels in its image. The process begins by calculating the number of pixels of widest part of the radius as shown in Figure 1 using the Pythagorean Theorem:

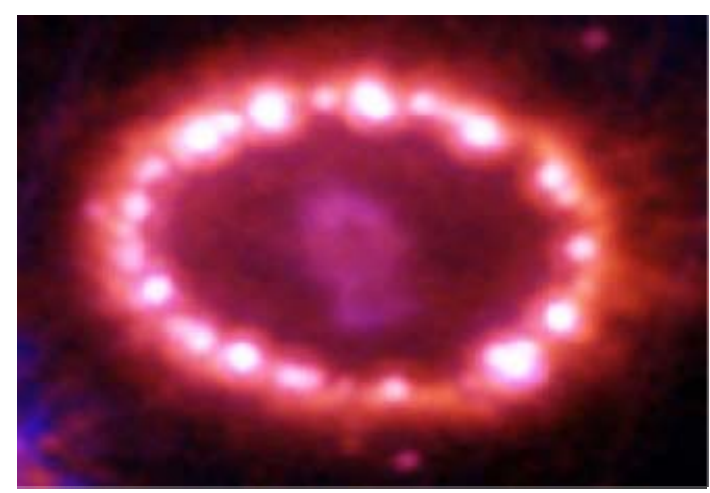

Figure 1. The image of SN 1987A as observed by HST in the year 2003 [16].

$$
\text { Size in Pixels }=\sqrt{\left(x-x_{1}\right)^{2}+\left(y-y_{1}\right)^{2}}
$$

Then convert this linear diameter to an angular size $(\theta)$ by multiplying this linear diameter by the image scale as follow:

$$
\theta=\text { size in pixels } \times \text { image scale }
$$

The image scale differs from one telescope to another since it depends on the properties of the CCD camera and the focal length of the telescope. For example for HST the image scale is 0.025 arc seconds per pixel [17] while for Chandra space telescope is 0.5 arc seconds per pixel [18]. In our case for SN 1987A the image scale is equal to 0.025 arc seconds per pixel, since the image has been taken from HST as shown in Figure 1. When the angular size has been measured, the actual size of the ring in kilometers can be calculated by using the derivation of the small angle formula which is given by [17]:

$$
d=(D \times \theta) / 206265^{\prime \prime}
$$

where $d$ is the true linear diameter of the ring, $D$ is the distance to the object, and 206265" is the number of arc seconds per one radian.

As the radius of supernova remnant has been calculated, the other physical parameters such as the expansion velocity, current mass, and the temperature can be obtained by using the following equations:

1) The expansion velocity of the remnant $\left(v_{\exp }\right)$, simply given by [19]:

$$
v_{\exp }=\frac{r}{t}
$$

where $r$ is the radius of the remnant which has a value about half the value of $d$, and $t$ represent the age of the remnant in seconds.

2) The current mass of the remnant $\left(m_{3}\right)$ [19]:

$$
m_{3}=m_{1}+m_{2}
$$

where $m_{1}$ represents the ejected mass from the explosion, $m_{2}$ represents the mass that swept from the surrounding medium and add to the ejecta and is given by [20]:

$$
m_{2}=\frac{4}{3} \pi \rho r^{3}
$$

where $\rho$ is the mass density of the interstellar medium.

3 ) The temperature of the remnant $\left(T_{\text {emp }}\right)$ [21]:

$$
T_{\text {emp }}=\frac{3 \mu m_{H}}{16 K} v_{\text {exp }}^{2}
$$

where $\mu$ represents the mean molecular weight, $m_{H}$ is the hydrogen mass for one atom, and $k$ is the Boltzmann constant.

In addition to that, this method can be also used to find the inclination angle of the system to our line of sight $i$ 
and this is done by taking the ratio of the smallest to the largest width of the ring as follow [17]:

$$
i=\sin ^{-1} \text { (smallest width/largest width) }
$$

In addition to the counting pixels method two theoretical models have been taken into account in order to make a comparison with them which are: Povida Model and Self-Similar Solution Model. The first model based upon the cooling process that occurring in the ejecta due to the synchrotrons radiation which results from charged particles (electrons, protons) that move around the line of the magnetic field with speed near the speed of light and this model is depend basically on the law of conservation of energy since the supernova remnant begin to decelerate as it expands far from the center of the supernova explosion as a result of increasing the sweeping mass from the surrounding medium [22] while the second model which is the Self-Similar Solution model is based upon the similarity between the explosion of the atomic bomb in the Earth's atmosphere and the explosion of a supernova in the interstellar medium and it study the structure of the remnant of the supernova explosion as a function of time and density and it provide the physical properties of the gas behind a strong spherical shock wave that formed by an explosion in an infinite medium with a density $\rho$ as a function of time, $t$, and radial position, $R$ [23] and this parameters considers as an important parameters in our case for SN 1987A.

\section{Results and Discussion}

The main goal of this study is to measure the expansion rate of the remnant of SN 1987A and follow its evolution. So in order to investigate this expansion the optical images of SN 1987A from 1995 to 2010 had been taken from HST these images are up to the year 2006 while those for the year 2007 and 2010 which are the only available images were taken from literature (Figure 2

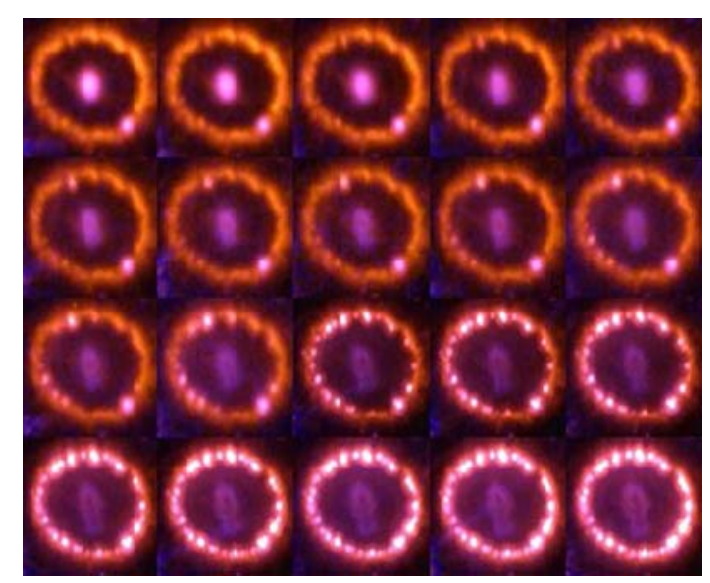

Figure 2. The image of SN 1987A as observed by HST from 1995 to 2006 [16].
[16]). Then the counting pixels method has been applied on it in order to measure the size parameter $d$ that uses to characterize this behavior which has been converted into physical size units by using the distance to SNR 1987A $51 \pm 1.2 \mathrm{kpc}[24]$, the results of applying this method are shown in Figure 3.

From the results that shown in Figure 3 it's noticed that, the expansion rate of the remnant is increasing from $0.21 \mathrm{pc}$ to $0.39 \mathrm{pc}$ between 1995 and 2010 and this expansion do not increase linearly all over as has been expected, but its increasing is shallowly exponentially, and this is due to the high density of the region that surrounds the exploding system (the equatorial ring) which works on decelerating the blast wave from expanding further. Besides that, when a comparison had been made between the results of this method and the results of the two other theoretical models which are: Povida model and the Self-Similar Solution model it's found that, there is a good agreement between the results of the Counting Pixels method and the results of the other two theoretical models about the size of the remnant and specially with the result of similarity solution as shown in Figure 4.

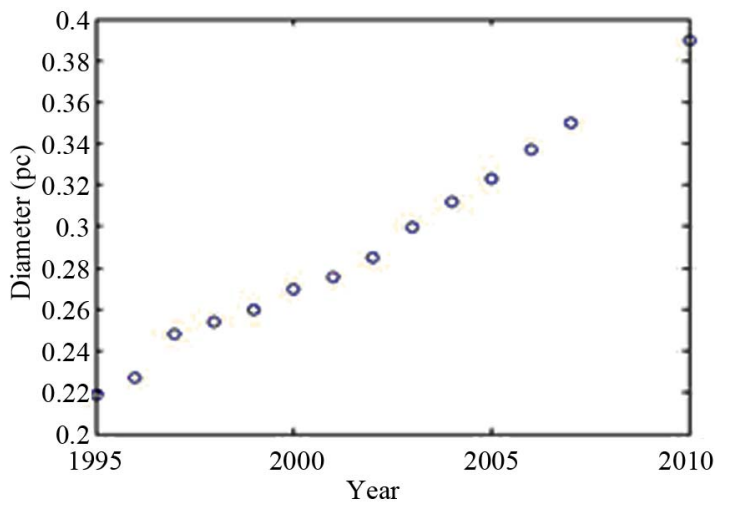

Figure 3. Radial expansion measure from the fits to the counting pixels method from 1995 to 2010.

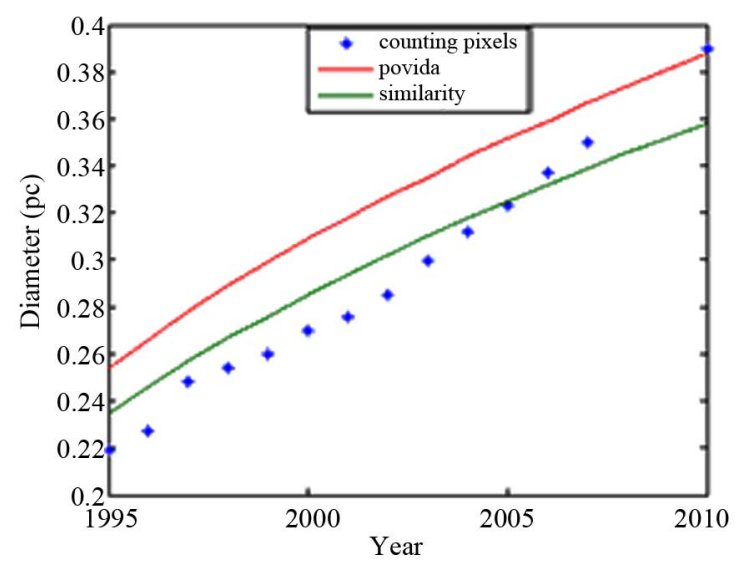

Figure 4. The results of radial expansion of SN 1987A that obtained from counting pixels method, povida model, and the Self-Similar Solution from 1995 to 2010. 
Although the counting pixels method shows agreement with similarity solution but is not very good agreement as has been expected since the later is deal with a uniform medium surround the explosion which is not the case of SN 1987A since its surrounded by ununiform medium that consists of nodes of mater that eject from the progenitor star in the later stage as red and blue supergiant wind that represented by the triple ring system. Furthermore, the counting pixels method deals with the images of supernova only at any circumstances which means its deal with the real situation of the case and not supposing any condition as other models do.

Moreover, after the size parameters has been available the other physical parameters can be calculated such as the expansion velocity of the remnant which is found to be decelerate from $3900 \mathrm{~km} \cdot \mathrm{s}^{-1}$ to $1200 \mathrm{~km} \cdot \mathrm{s}^{-1}$ during the past 23 years from the explosion and this result shows a plausible agreement with the results of Racusin et al. which was found to be $1700 \mathrm{~km} \cdot \mathrm{s}^{-1}$ in 2004 , Parker et al. which was found to be $1412 \pm 354 \mathrm{~km} \cdot \mathrm{s}^{-1}$ in 2007 , and the results of the Hydrodynamic models which indicate the forward shock velocity is dramatically decelerate from $\sim 3500$ to $\sim 1000-1500 \mathrm{~km} \cdot \mathrm{s}^{-1}$ after day 6000 from the explosion and afterwards [24].

In addition to that, the Equations (5) and (7) have been used to calculate the current mass and the temperature of the remnant which are found to be equal to $11.8 \mathrm{M}_{\odot}$, and $3.3 \times 10^{8}{ }^{\circ} \mathrm{K}$ respectively. Table 1 lists the results of some physical parameters of SN 1987A that obtained from Counting Pixels Method in comparison with that obtained from Povida Model and Self-Similar Solution Model.

\section{Conclusion}

It's found that, the high density of the region that surround the exploding system which represent by the

Table 1. The results of some physical parameters of SN 1987A by applying counting pixels method in comparison with other models.

\begin{tabular}{ccccc}
\hline Quantity & Unit & $\begin{array}{c}\text { Povida } \\
\text { model [25] }\end{array}$ & $\begin{array}{c}\text { Self-similar } \\
\text { solution[25] }\end{array}$ & $\begin{array}{c}\text { Counting } \\
\text { pixels } \\
\text { method }\end{array}$ \\
\hline $\begin{array}{c}\text { Expansion } \\
\text { velocity }\left(v_{2}\right) \\
\text { Current } \\
\text { mass }\left(m_{3}\right)\end{array}$ & ${\mathrm{km} \cdot \mathrm{s}^{-1}}_{\odot}$ & 1328 & 1220 & 1200 \\
$\begin{array}{c}\text { Diameter } \\
(d)\end{array}$ & $\mathrm{pc}$ & 0.391 & 0.362 & 11.2 \\
$\begin{array}{c}\text { Age }(t) \\
\begin{array}{c}\text { Temperatur } \\
\mathrm{e}\left(T_{\mathrm{emp}}\right)\end{array}\end{array}$ & $\mathrm{year}$ & 23 & 23 & 0.39 \\
$\begin{array}{c}\text { The rate of } \\
\text { expansion }\end{array}$ & /yr & 0.111 & 0.139 & 0.141 \\
\hline
\end{tabular}

equatorial ring play a significant role in decelerating the blast wave of supernova from expanding further and on decelerating the radial expansion from $3900 \mathrm{~km} \cdot \mathrm{s}^{-1}$ to $1200 \mathrm{~km} \cdot \mathrm{sec}^{-1}$ during the past 23 years, and with current size equal to $0.39 \mathrm{pc}$. Moreover, the current measurements of the sweeping mass of the remnant and its temperature indicate that, the remnant is still in the free expansion phase since the sweeping mass is still smaller than the ejected mass (which is equal $8.8 \mathrm{M}_{\odot}$ ) and the temperature of the remnant is still larger than $10^{6}{ }^{\circ} \mathrm{K}$ which is the temperature of the remnant when its enter the second evolutionary stage which is called the adiabatic expansion phase. So it can be concluded that, the counting pixels method has a good agreement with self-similar solution model results especially when dealing with the estimation of the size and expansion rate of type II supernova.

\section{REFERENCES}

[1] J. E. Van Zyl, "Variable Stars VI," The Astronomical Society of Southern Africa, Johannesburg Centre Monthly Newsletter, 2003.

[2] J. C. Wheeler, "Cosmic Catastrophes: Exploding Stars, Black Holes, and Mapping the Universe," 2nd Edition, Cambridge University Press, New York, 2007, pp. 79-135. doi:10.1017/CBO9780511536625.007

[3] W. D. Arnett, "On the Early Behavior of Supernova 1987A," Astrophysical Journal, Vol. 331, No. 1, 1988, pp. 377-387. doi:10.1086/166564

[4] S. Park, D. Burrows, G. Garmire, R. McCray, J. Racusin and S. Zhekov, "Chandra Observations of Supernova 1987A," Astrophysical Journal, Vol. 937, 2007, pp. 4350.

[5] S. Jogee, "Introduction to Astronomy," 2006. http://www.as.utexas.edu/ sj/a301-fa06/lec2-3.partial.pdf

[6] R. Wilson, "Astronomy through the Ages: The Story of the Human Attempt to Understand the Universe," Taylor \& Francis e-Library, London, 2005.

[7] W. D. Arnett, R. Bahcall, J. Kirshner and S. Woosley, "SUPERNOVA 1987A," Annual Review of Astronomy and Astrophysics, Vol. 27, 1989, pp. 629-700. doi:10.1146/annurev.aa.27.090189.003213

[8] B. W. Carroll and D. A. Ostlie, "An Introduction to Modern Astrophysics," 2nd Edition, Pearson Education, San Francisco, 2007, pp. 524-542.

[9] R. Roger and T. Landecker, "Supernova Remnants and the Interstellar Medium," Cambridge University Press, Cambridge, 1988, pp. 12-20.

[10] R. Schaeffer, "SN 1987A. A Review," Acta Phsica Polonica, Vol. 12, 1990, pp.357-376.

[11] B. J. Becker, "Eclecticism, Opportunism, and the Evolution of a New Research Agenda: William and Margaret Huggins and the Origins of Astrophysics," Ph.D. Thesis, University of California, Oakland, 1993.

[12] B. M. Gaensler, "Barrels, Jets and Smoke-Rings: Under- 
standing the Bizarre Shapes of Radio Supernova Remnants," Ph.D. Thesis, University of Sydney, Sydney, 1999.

[13] E. J. Wampler and A. Richichi, "Observations of Nebular Emission Lines towards SN 1987A," Astronomy and Astrophysical Journal, Vol. 217, No. 1-2, 1989, pp. 31-34.

[14] A. Tziamtzis, P. Lundqvist, P. Gröningsson and S. Nasoudi-Shoar, "The Outer Rings of SN 1987A?" Astrophysical Journal, Vol. 527, 2010.

[15] E. N. Michle, "The Structure and Development of Supernova Remnant 1987A," Ph.D. Thesis, University of Colorado, Colorado, 1998.

[16] K. E. Kjær, "Integral Field Spectroscopy Observations of SN 1987A," Ph.D. Thesis, University of Munich, Munich, 2007.

[17] J. Kolena, "Chandra/ds9 (the Windows Version) Faq Page," 2009. http://www.phy.duke.edu/ kolena/ds9_faq.html.

[18] S. Palen, "Properties of Supernova," 2000. http://www.astro.washington.edu/courses/labs/clearingho use/labs/Propsn/propsn.html

[19] R. Al-Sarraf, "New Model for SN 1987A,” MSc. Thesis,
University of Baghdad, Baghdad, 1990.

[20] T. T. Arny, "Exploration: An Introduction to Astronomy," 2nd Edition, McGraw-Hill Companies, New York, 1998, pp. 390-391.

[21] H. Karttunen, P. Kröger, H. Oja, M. Poutanen and K. Donner, "Fundamental Astronomy," 5th Edition, SpringerVerlag, New York, Berlin, Heidelberg, 2007, pp. 107-224. doi:10.1007/978-3-540-34144-4

[22] A. Povida, "Nova, Novides, ET. Supernovae," France: Centre National de la Recherche Scintifique, Vol. 121, 1964, pp.221-230.

[23] J. Lequeux, "The Interstellar Medium," Springer-Verlag, Berlin, Heidelberg, 2005, pp. 275-285.

[24] J. L. Racusin, S. Park, S. Zhekov, D. Burrows, G. Garmire and R. McCray, "X-Ray Evolution of SN 1987A The Radial Expansion," The Astrophysical Journal, Vol. 703, No. 2, 2009, pp. 1752-1759. doi:10.1088/0004-637X/703/2/1752

[25] L. T. Ali, "Theoretical Study of the Physical Parameters of the Supernova 1987A," MSc. Thesis, University of Baghdad, Baghdad, 2011. 